\title{
Research on the Countermeasures of Building Smart Community Based on Cloud Computing
}

\author{
Liu Lijuan \\ College of Information Engineering, Nanjing Normal University Taizhou College \\ JiChuan East Road No. 96, Hailing District, Taizhou, Jiangsu, China
}

\begin{abstract}
:
Smart communities are the result of the evolution of communities under the development of new technologies. Many cities regard smart communities as one of the three regional demonstrations of smart city construction. Based on the experience of the construction of excellent intelligent communities at home and abroad, this paper analyzes the problems and difficulties in the current smart community construction, puts forward specific feasible countermeasures, and designs the smart community structure, which can promote the humanization of community governance, the integration of community services and the intelligentization of home life.
\end{abstract}

Keywords: smart community; cloud computing; J2EE

\section{Introduction}

With the deepening of the construction of a global smart city, the smart community, as one of the most close to the concept of citizen life, is increasingly valued by local governments. In 2012, China's smart communities started in Beijing and Shanghai, and started late. In the early stage of the construction of smart communities, the investment was large, and it was basically led by the government. It was easy to produce a series of problems such as insufficient vitality, shortage of funds, and separation from the living needs of residents. In the process of building a smart community, it is necessary to carry out certain research and exploration, improve the level of construction, point out the direction for the wisdom of the community, promote the excellent experience, and strive to create a new type of community with residents and communities community wisdom, and government efficiency.
1. Experience in building smart communities at home and abroad

The basic unit of a smart city is a smart community. In recent years, some foreign countries have rich experience in smart community governance, while China's smart communities are still in the stage of imitation and exploration, and the development of different regions is different.

The Bristol Smart Community in the UK integrates the data of the Community Monitoring Center with the database of the City Monitoring Center to provide residents with data outside of their personal privacy. Residents can use the mobile APP to check the weather conditions of the road, weather and garbage trucks in real time. . Bristol has made strong guidance to the smart community through the city council, and with the active participation of universities, citizens, social organizations, etc., the construction of the Bristol Smart Community is very successful, reasonable, 
rigorous and scientific.

Singapore's smart community has been at the forefront of the world. Since 1992, smart city construction has begun. In the smart community, residents can inquire about book information, apply for tax relief, understand supermarket price, etc., it can provide better services to residents through mechanisms such as mutual participation, sensor data transmission, and effective information sharing.

The smart community of Guangnei Street in Xicheng District, which was started in Beijing in 2011, integrates public services, community management and mutual assistance services on the public service platform with government-led, corporate, social and market operations, which greatly facilitates Daily life of residents.Beijing has released three core indicators of smart communities: infrastructure, community services and community management, using infrastructure platforms to innovate community management and improve service capabilities.

The Lujiazui Smart Community Experience Center, which was built in Shanghai in 2015, has built a community public service information platform and developed application systems such as electronic bulletin boards, smart navigation, and intelligent parking services.Using the database to summarize all the public information of the community, residents can use a card that collects multiple card information to achieve many applications.

The success of the construction of excellent smart communities at home and abroad is inseparable from the scientific and rational overall planning, the comprehensive cooperation of all parties from the society, the sharing of information, the extensive participation of residents and the perfect guarantee system.

\section{Problems and difficulties in the construction of smart communities}

Promoting the high-quality development of the city is at the forefront, and we must thoroughly implement the strategic deployment of the country to promote smart cities, "Internet +", big data, and accelerate the construction of smart cities. At the same time, we will build a smart community, promote the extension of public services of various departments to the community through informationization, encourage application integration and model innovation of various life services, and promote online and offline interactions for community services. Encourage community property to connect and interact with community-related management platforms through information technology, and gradually implement intelligent applications in the fields of community personnel management, security monitoring, and parking services. Innovate the intelligent service model of family life through the promotion of smart home applications. Do a good job in the demonstration of smart communities and encourage mature smart community models to be promoted throughout the city.

In the process of building a smart community, the following problems exist:

(1) The degree of data integration and utilization is low. Inter-departmental information barriers are serious, data management is scattered, data collection and maintenance, sharing standards are not uniform. There are problems such as unclear of data responsibilities 、 ownership and sharing openness a and processes.Data-based socialization is low-level and cannot fully exploit data dividends.

(2) The overall planning of the platform needs to be strengthened. The coordination of various common business platforms is not strong, the level of business development and application is not high, and there are cases of repeated construction. There is no forward-looking and holistic top-level design and planning for information system construction. The overall architecture lacks flexibility and scalability.

(3) The construction promotion model needs to be enriched. The sources of funds for information construction are relatively simple, the amount of social capital participation is insufficient, there is no sustainable investment operation mode, and it is impossible to support the information construction and operation and maintenance of various departments.

(4) The supporting mechanism system needs to be improved. The structure and quantity of professional and technical personnel cannot meet 
the needs of informatization development. The standards and norms of project construction, assessment and assessment, data resource coordination and other aspects have yet to be formulated and improved. The system and mechanism for promoting the construction of smart communities need to be optimized.

(5) Information security protection needs to be strengthened. Data security is seriously threatened, lacking standards for data desensitization and decryption, log tracking of data flow, and development of standards and specifications for data.

\section{Suggestions for the development of smart communities}

As the last mile of smart city construction, the smart community includes family planning, livelihood security, and convenient travel within a specific scope. In the process of construction, it is necessary to implement the plan in stages, and to optimize the lifestyle of the residents.

(1) Analysis of needs, each performing their duties

The construction of a smart community requires first understanding the needs orientation of the government, enterprises, community neighborhood committees and residents, and mobilizing all parties to participate. The government is the instructor of the construction of smart communities. It is necessary to expand the depth and breadth of the scope of data, better serve the people, improve the efficiency of work, improve the image of serving the people, and governing for the people. As a paid service provider, enterprises need to understand the various needs of the community residents in order to better provide personalized services, improve enterprise efficiency, and enhance corporate competitiveness. As the manager of the smart community, the community neighborhood committee needs to use the database and management platform to handle daily affairs more conveniently, serve the people conveniently, and provide a more comfortable community life. As the most direct users and participants of the smart community, residents need to experience the smart security, real-time monitoring, information consultation and other services brought by the smart community. Only by understanding the actual needs of the government, enterprises, neighborhood committees, and residents can we build a better platform and maximize benefits.

(2) Overall planning and standard setting

As a systematic project, the construction of smart communities should be extensively researched, coordinated, and consulted in the early stage, and scientific indicators should be formulated so that their construction can be continuously improved, updated and developed in the course of practice.

According to the guidelines of the Ministry of Housing and Urban-Rural Development on the construction of smart communities and the excellent experience at home and abroad, we will develop assessment indicators and assessments based on documents such as national economic and social development, smart city construction, and the characteristics of enterprises, the scale of social organizations, and the needs of residents. Mechanism to improve the overall construction of the project. Strengthen the tracking analysis, supervision and inspection of the implementation progress and quality, and establish a target responsibility system and a responsibility assessment system. Through the third-party organization to carry out quality tracking of the whole process of engineering construction, including engineering consulting, supervision, design, acceptance testing, effectiveness evaluation, etc., to create a full-cycle management and control system that can achieve planning, comprehensive evaluation, planning execution, performance evaluation.

(3) Design framework, distribution and construction

The overall architecture of the smart community can be divided into several parts: the infrastructure layer, the integrated service platform layer, and the smart community application layer. The infrastructure construction includes the facility layer as the carrier, the network layer to realize the interconnection of various networks, and the real-time information transmission sensing of the sensing layer. The integrated service platform layer integrates existing government affairs, 
transportation, medical care, education and other database information to form social management, community service, interactive communication, information consultation and other modules to provide a friendly user interface. The smart community application layer is designed for users to provide projects such as property management, convenience services and community governance. The government, enterprises, neighborhood committees and residents can all achieve final access.

(4) Consolidate the foundation and strengthen security

Establish an information security system, take prevention as the main task, combine management and control, implement emergency linkage, conduct regular information system security inspections, focus on important information systems and cloud computing centers, organize emergency plan preparation and drills, and improve information security emergency response capabilities. Strengthen the construction of network security protection system. Adhere to the importance of both network protection and information resources, external and internal prevention, timely adjust the level of information disclosure and confidentiality, achieve a balance between information confidentiality and information openness, and establish a comprehensive information security management system.

(5) Distribution implementation, strong promotion

The construction of a smart community has a long way to go. The government, enterprises, and residents can provide different resources. Based on the existing conditions, it is recommended to adopt a distributed approach to implement the pilot phase, the ascending phase, the promotion phase and the maturity phase. In the pilot phase, the "government-led" model was adopted. The government coordinated the overall situation, established a work coordination mechanism, and strengthened the overall design guidelines and normative constraints. However, there were many problems such as insufficient innovation, insufficient development momentum and large financial pressure. In the rising stage, the "enterprise-led" model is adopted. The rights are decentralized by the government, and all kinds of enterprises compete for opportunities. All kinds of emerging enterprises compete to generate and continuously improve market competitiveness through innovation. The market vitality is highlighted, but there are difficulties in resource integration in the later period. There are many problems such as the decline in market credibility and market failure. In the promotion phase, the government adopts the model of "government guidance and market operation". The government implements each piece of the project to authorize specific enterprises, and the government gives policy and financial support. This model not only ensures the unified deployment of the government, but also exerts the vitality of the market economy. But in the final stage of maturity, the shortcoming is that it is easy to ignore the will of people's livelihood.

The final stage of maturity adopts the "big integration" model, so that more residents, social groups and third institutions can join the construction of smart communities, fully embodying the wisdom of people-oriented, professional service, high efficiency and enjoy quality life. The original intention of social construction.

(6) Emphasis on talents and create an atmosphere

Make use of resources such as local higher education, increase the popular technology of Internet of Things, cloud computing, big data and engineering project management training in smart community applications, and promote the transformation of regional education industry to "production, study and research". Organize systematic, normalized, customized training and lectures in government departments and enterprises, promote knowledge update of government personnel and enterprise staff, organize technical seminars, hold innovation and entrepreneurship contests, and provide smart city construction talents' soil.

\section{Smart Community Construction Architecture} Design

Based on the existing principles of resource 
integration, business drive, adaptive integration and expansion, according to the construction goals of the smart community, the external environment, internal composition and mutual relationship of the smart community are designed to form the overall community structure as shown in figure 1 .

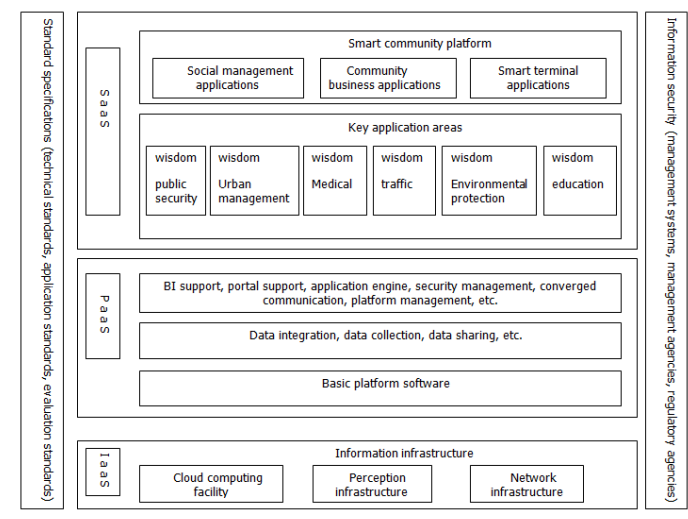

Figure 1: Overall architecture of smart community

\section{(1) Internal composition}

From the top down, the smart community consists of three layers: the application layer (SaaS), the computing and integration and exchange (PaaS), soft platform support layer (IAAS). Among them: The SaaS layer provides applications for smart communities. Including: Multiple portals for smart communities, community services, community management, etc. Community platform users can order corresponding portals and applications. The PaaS layer supports component open, third-party application access. Including: providing BI support class, application engine class and other component services for SaaS function development; using data storage layered to achieve data application and sharing; ESB to achieve integration of platform internal and external applications. The operating system, database, and middleware are all the basic software included in this layer. IaaS is deployed on a cloud platform that includes various infrastructures to provide access to smart communities to property and residents.

(2) External environment

The relationship between the smart community and the external environment is: Data exchange between the smart community and the national basic data system, the telecommunications system provides network access and information communication for the smart community, and provides application access for other projects in the smart city.

\section{Conclusion}

In the information age, the smart community is the key point and basic unit of smart city construction. This paper draws on the experience of the construction of excellent smart communities at home and abroad, analyzes the practical problems of urban smart community construction in China, and proposes countermeasures for phased implementation. There is a long way to go, and we need to constantly develop and innovate, explore the way forward, and embark on a road of intelligent community construction that is in line with national conditions.

\section{References}

[1] Wang Lingqun, He Shizhen, Yuan Xiaohua, Zhang Shutai, "Intelligent Community Architecture Design Based on J2EE and Cloud Computing", Laboratory Research and Exploration,pp.123-127, 2014.

[2] Xu Dacheng, Research and Implementation of Intelligent Campus Information System Based on Internet of Things and Cloud Computing, Shaanxi: Xi'an University of Electronic Science and Technology, 2015.

[3] Zhu Yiying, Research on the construction of smart communities in Tongzhou District of Nantong City, Shanghai: Donghua University, 2017.

[4] Lu Wei, Research on the Countermeasures for the Construction of Wisdom Community in Wuxi City, Gansu: Northwest Normal University, 2017.

[5] [5] Wang Zhaoqing, He Yong, "Research on the construction of smart city based on big data cloud platform", Intelligent Processing and Application, pp. 87-90, 2017.

[6] Liu Nan, Path selection of smart city construction under the background of informationization, Shanxi: Xi'an University of Architecture and Technology, 2016.

[7] Liu Yang, Problems in the construction of smart cities in Jinan and countermeasures, Shandong: Shandong University, 2018. 


\section{About the Author}

Liu Lijuan received a master's degree from Nanjing University of Aeronautics and Astronautics, where she was an assistant in Nanjing Normal University Taizhou College (2008) 、 lecturer (2010) and Associate Professor(2018). Her research interests include cloud computing, big data theory and practical application. 